\section{Kompetenzorientierte Bachelor- und Masterstudien}

\section{Ein Einblick in aktuelle Entwicklungen an Schweizer Fachhochschulen}

Vor zehn Jahren wurde in der Schweiz mit der Eröffnung der ersten Fachhochschulen ein neuer Hochschultypus geschaffen und mit einem Leistungsauftrag für Aus- und Weiterbildung, anwendungsorientierte Forschung und Entwicklung und Dienstleistungen für Dritte betraut. Nach der Aufbauphase war die Frage, wie die bildungspolitische und -theoretische Diskussion an den Fachhochschulen umgesetzt werden kann. Zwei ausgewählte Entwicklungen am Beispiel der Fachhochschulen für Soziale Arbeit in der Schweiz.

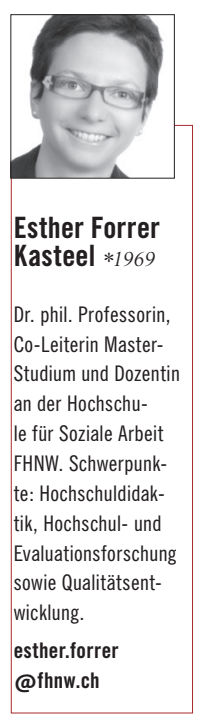

1995 wurde vom eidgenössischen Parlament das Bundesgesetz über die Fachhochschulen verabschiedet. Auf dieser Grundlage leitete der Bund die Umgestaltung der in seine Zuständigkeit fallenden Bildungsangebote der Tertiärstufe zu Fachhochschulen für Technik, Wirtschaft und Gestaltung ein. Für jene Ausbildungsangebote, welche zum damaligen Zeitpunkt noch der Regelungskompetenz der Kantone unterstanden - etwa jene der Sozialen Arbeit - wurde mit kantonalen Fachhochschulgesetzen, die weitgehend entlang der Bundesgesetzbestimmungen formuliert wurden, ebenfalls die rechtlichen Grund- lagen für deren Aufbau geschaffen. Mit dem revidierten Fachhochschulgesetz, das 2005 in Kraft getreten ist, obliegt die Verantwortung über die Fachhochschulen aller Fachbereiche dem Bund (vgl. Gredig und Truniger 2005).

Gemäss Artikel 2 des revidierten Fachhochschulgesetzes (Stand Juni 2006) sind Fachhochschulen Ausbildungsstätten der Hochschulstufe, die grundsätzlich auf einer beruflichen Grundausbildung aufbauen. Mit dem neuen Hochschultypus - den praxisorientierten Diplomstudien - sollen Studierende „... auf berufliche Tätigkeiten vorbereitet werden, welche die Anwendung wissenschaftlicher Erkenntnisse und Methoden sowie, je nach Fachbereich, gestalterische und künstlerische Fähigkeiten erfordern“ (vgl. Artikel 2 des Fachhochschulgesetzes). In Artikel 3 wird explizit festgehalten, dass die Fachhochschulen einen vierfachen Leistungsauftrag zu erfüllen haben: Sie sollen Diplomstudiengänge führen, diese durch ein Angebot an Weiterbildungsveranstaltungen ergänzen, anwendungsorientier- te Forschungs- und Entwicklungsarbeiten durchführen und Dienstleistungen für Dritte erbringen. Gegenwärtig werden in der Schweiz sieben öffentlich-rechtliche Fachhochschulen und eine private Fachhochschule geführt, sechs in der deutschsprachigen Schweiz, eine in der französischsprachigen und eine in der italienischsprachigen. Ausser an der Fachhochschule Kaleidos werden an allen Fachhochschulen Bachelor-Studien in Sozialer Arbeit geführt.

Ein bedeutsamer Treiber der Entwicklung der Fachhochschulen in der Schweiz ist die Bologna-Reform. Mit der 1999 unterzeichneten Erklärung von Bologna verpflichtete sich auch die Schweiz, ihre Ausbildungen im hochschulischen Tertiärbereich den europäischen Vorgaben anzupassen und damit wichtige Neuerungen, wie beispielsweise die Einführung eines gestuften und modularisierten Studiensystems (Bachelor und Master) sowie die geforderte Kompetenz- bzw. Outcomeorientierung, umzusetzen. Der in der Bologna-Reform geforderte Entwicklungsprozess ist derzeit in der Schweiz noch in vollem Gange und soll bis 2010 abgeschlossen sein.

\section{Aktuelle Entwicklungen}

Werden nun die aktuellen Entwicklungen an den Schweizer Fachhochschulen in Sozialer Arbeit beleuchtet, fällt auf, dass die Umsetzung der geforderten Kompetenzorientierung der Studiengangsentwicklungen sowie die bildungspolitische Debatte rund um die Einführung von Master-Studien ab Herbstsemester 2008/2009 die Diskussion prägen. Soll nämlich die in der Bologna-Reform geforderte Kompetenzorientierung nicht nur Ideal bleiben, sondern konsequente Umsetzung erfahren, muss pro Studiengang

Nutzen Einblick in den aktuellen Stand der kompetenzorientierten Studiengangsentwicklung sowie der Konzipierung von konsekutiven Master-Studien in der Schweizer Fachhochschullandschaft in Sozialer Arbeit.

Das Wichtigste in Kürze In der Schweizer Fachhochschullandschaft der Sozialen Arbeit werden gegenwärtig kompetenzorientierte Studiengänge entwickelt und konsekutive Master-Studien konzipiert. Diese beiden Entwicklungen stehen zehn Jahre nach der Eröffnung der ersten Fachhochschulen in der Schweiz im Brennpunkt bildungspolitischer und bildungstheoretischer Diskussionen. 
ein verbindliches Kompetenzprofil entwickelt und die Umsetzung der beiden Stufen realisiert werden.

\section{Kompetenzprofil als Kernstück}

Bis heute bleiben im Zusammenhang mit der Umsetzung der Kompetenzorientierung noch viele Fragen unbeantwortet. Dies ist unter anderem darauf zurückzuführen, dass es bislang nicht gelungen ist, sich auf eine theoretisch fundierte und allgemein akzeptierte Kompetenzdefinition zu einigen (vgl. Vonken 2005: S. 11).

Da für die Bachelor-Konzepteingabe von allen Fachhochschulen die Entwicklung von Kompetenzprofilen eingefordert wurde, liegen in der Schweiz solche für sämtliche Bachelor-Studien vor (vgl. Forrer/Wilhelm 2006: S. 25). Darüber hinaus hat die Fachkonferenz Soziale Arbeit ein fachhochschulübergreifendes, disziplinenspezifisches Kompetenzprofil erarbeitet. Dieses wurde im Zusammenhang mit der Master-Konzepteingabe beim Bund zu einem gestuften Kompetenzprofil weiterentwickelt und in ein gemeinsam erstelltes Rahmenkonzept für Masterstudien in Sozialer Arbeit in der Schweiz (vgl. SASSA 2007A) integriert. Die Umsetzung des Rahmenkonzeptes, insbesondere des darin festgehaltenen Kompetenzprofils, wurde von den Verantwortlichen aller Schweizer Fachhochschulen in Sozialer Arbeit durch die Unterzeichnung eines „Letter of Intent" verbindlich geregelt.

Mit welcher Fundierung und Ernsthaftigkeit die einzelnen Fachhochschulen ihr eigenes gestuftes Kompetenzprofil generierten, lässt sich am Beispiel der Hochschule für Soziale Arbeit der Fachhochschule Nordwestschweiz verdeutlichen. Hier wurde ein dreistufiges Kompetenzprofil (Bachelor, Master und $\mathrm{PhD}$ ) sowohl theorie- als auch empiriebasiert entwickelt. Theoriebasiert, indem zum einen der Kompetenzbegriff verbindlich definiert und zum andern in die Bestimmung der Kompetenzen sowohl bildungsals auch gesellschaftstheoretische Grundlagen einbezogen wurden. Empiriebasiert wiederum, indem das entwickelte Kompetenzprofil anhand von vier intern und extern durchgeführten Studien in einem iterativen Verfahren fortlaufend weiterentwickelt wurde. In allen Untersuchungen wurde deutlich, dass die Zielgruppen das Kompetenzprofil als gut bis sehr gut beurteilen. Zudem sind sie überzeugt, dass die gewählten Kompetenzen grundlegend dafür sind, dass künftige AbsolventInnen für die immer komplexeren Aufgaben im Berufsfeld der Sozialen Arbeit adäquate Lösungen erarbeiten können (vgl. Forrer Kasteel/Markwalder/Parpan-Blaser/ Wilhelm 2007).

\section{Konsekutive Master-Studien}

In der Schweizer Fachhochschullandschaft werden ab Herbstsemester 2008/09 erstmals konsekutive Master-Studien in unterschiedlichen Fachbereichen starten - zusätzlich zu den bereits laufenden konsekutiven Master-Studien in Architektur und Pädagogik. Im Unterschied zu exekutiven Master-Studien (Master of Advanced Studies, MAS) werden in konsekutiven Master-Studien generalistische und nicht spezifische, thematisch fokussierte Kompetenzen vermittelt. Ferner sind diese konsequent zweistufig aufgebaut und setzen einen Bachelor-Abschluss in Sozialer Arbeit voraus, während bei exekutiven Master-Studien Bachelor-Abschlüsse unterschiedlicher Studiengänge zugelassen sind.

Master-Abschlüsse gelten an den Universitäten als Regelabschlüsse, an den Fachhochschulen sollen sie hingegen nur

Literatur:

BBT (BUNDESAMT FÜR BERUFSBILDUNG UND TECHNOLOGIE) UND EDK

(SCHWEIZERISCHE KONFERENZ DER KANTONALEN ERZIEHUNGSDIREKTOREN) (2006)

Vereinbarung zwischen dem Bund und den Kantonen über den Aufbau von Master-Studiengängen

an Fachhochschulen. Erläuternder Bericht.

BUNDESAMT FÜR STATISTIK (2006)

Bildungsperspektiven. Studierende und Hochschulabsolventen: Szenarien 2006-2015. Neuenburg.

DIE BUNDESVERSAMMLUNG DER SCHWEIZERISCHEN EIDGENOSSENSCHAFT (6. ОKTOBER I999)

Bundesgesetz über die Fachhochschulen (Fachhochschulgesetz, FHSG).

vgl. http://www.admin.ch/ch/d/sr/4/414.71.de.pdf; letztes Download am 12.11.2007.

FORRER, ESTHER/ WILHELM, ELENA (2006)

Bachelor of the labour market. Professionally orientierted higher education.

Country report of Switzerland in the framework of the European Balama study. Nonpublished report.

FORRER KASTEEL, ESTHER/MARKWALDER, SONJA/ PARPAN-BLASER, ANNE/WILHELM, ELENA (2007)

Das Kompetenzprofil als Kernstück der Entwicklung des Masterstudiums Soziale Arbeit mit Schwer-

punkt Soziale Innovation. IN Soziale Innovation. Forschung und Entwicklung in der Sozialen Arbeit 2007.

Fachhochschule Nordwestschweiz. Hochschule für Soziale Arbeit, S. 46-68.

GREDIG, DANIEL (2005)

The Co-Evolution of Knowledge Production and Transfer. Evidence-based Intervention Development as an approach to improve the impact of evidence on Social Work Practice. In: Sommerfeld, Peter (Hrsg.) Evidence based Social Work - Towards a New Professionalism? Bern/Berlin/Bruxelles/Frankfurt am Main/New York/ Oxford/Wien, S. 173-199.

GREDIG, DANIEL/SOMMERFELD, PETER/ KÄGI, URS/ MÄDER, UELI/VERKUIL, ARIE HANS (2003)

Evidence-based Intervention Development. Programm outline. Brugg. Unpublished manuscript.

GREDIG, DANIEL/TRUNIGER, LUZIA (2005)

Die Ausbildung in Sozialer Arbeit in der Schweiz. Schlaglichter auf eine Bildungslandschaft im Wandel. IN Soziale Arbeit, H. 5-6, S. 208-217.

HOLLENSTEIN, LEA/ SOMMERFELD, PETER/BAUMGARTNER, EDGAR (2007)

Das Forschungsprogramm «Evidence-Based Intervention Development» (EBID)

Professionalisierung durch kooperative Wissensbildung. IN Soziale Innovation. Forschung und Entwicklung in der Sozialen Arbeit 2007. Fachhochschule Nordwestschweiz, Hochschule für Soziale Arbeit, S. 8-19.

SASSA (FACHKONFERENZ SOZIALE ARBEIT DER FH SCHWEIZ) (2007A)

Master in Sozialer Arbeit, Rahmenkonzept. vgl. http://www.sassa.ch/webserv/webmod/WebModuleDocsActions.cfm / Rahmenkonzept\%20SASSA\%2007\%2D05\%2Epdf?idcat=189\&iddoc $=1297$ \&etfile=1 \&lng=FR\&an onymous $=1$; letztes Download am 9.11.2007.

SASSA (FACHKONFERENZ SOZIALE ARBEIT DER FH SCHWEIZ) (2007B)

Bericht zur Nachfrage von Master-Studiengängen in Sozialer Arbeit auf dem Arbeits- und Bildungsmarkt in der Schweiz. Unveröffentlichter Bericht.

SOMMERFELD, PETER (2003)

Zukunftsszenarien Soziale Arbeit. Überlegungen zur Lösung sozialer Probleme. Aarau: Sonderdruck.

VONKEN, MATTHIAS (2005)

Handlung und Kompetenz. Theoretische Perspektiven für die Erwachsenen- und Berufspädagogik. Wiesbaden. 
von ausgewählten Studierenden erworben werden können. So wird gemäss erläuterndem Bericht zur Vereinbarung zwischen dem Bund und den Kantonen über den Aufbau von Master-Studien an Fachhochschulen davon ausgegangen, dass beispielsweise in den Bereichen Technik, Wirtschaft und Soziale Arbeit maximal $25 \%$ der Bachelor-Absolvierenden ein Master-Studium absolvieren, in den Bereichen Gesundheit und bildende Kunst hingegen $70 \%$ (vgl. BBT und EDK 2006: S. 11). Zudem wird festgehalten, dass mangels finanzieller und personeller Ressourcen öffentlich-rechtliche Fachhochschulen nur eine beschränkte Zahl von Master-Studien anbieten können (vgl. BBT und EDK 2006: S. 6).

Die Führung konsekutiver Master-Studien an öffentlich-rechtlichen Fachhochschulen bedarf der Bewilligung durch den Bund. Insgesamt reichten die Träger 86 Gesuche für Master-Studien mit Studienbeginn Herbstsemester 2008/09 ein, davon sind zahlreiche Gesuche als Kooperationsangebote konzipiert. Ende 2007 entschied der Bund darüber.

Für die Disziplin der Sozialen Arbeit wurden die beiden folgenden Studien bewilligt:

- „Master of Arts in Sozialer Arbeit mit Schwerpunkt Soziale Innovation“ der Fachhochschule Nordwestschweiz

- "Master of Science in Social Work“ der Fachhochschulen Ostschweiz, Zürich, Zentralschweiz und Bern

Für die französischsprachige Schweiz ist ein weiteres Master-Studium in Sozialer Arbeit auf einen späteren Zeitpunkt in Planung, für die italienischsprachige Schweiz ist die Entwicklung eines MasterStudiums noch weitgehend offen.

Das Master-Studium „Master of Arts in Sozialer Arbeit mit Schwerpunkt Soziale Innovation" ist international ausgerichtet und wird von der Fachhochschule Nordwestschweiz in enger Kooperation mit der Evangelischen Fachhochschule Freiburg im Breisgau sowie in Zusammenarbeit mit der Universität Basel angeboten. Die- ses Studium zeichnet sich durch Anwendungsorientierung, Forschungsbasierung und Internationalität aus. Damit diese drei Forderungen nicht nur Programmatik bleiben, sondern konkrete Umsetzung erfahren, wurde das Master-Konzept bewusst theoretisch-empirisch und konsequent kompetenzorientiert konzipiert. Theoretisch untermauert wird dieses Studium durch das an der Hochschule für Soziale Arbeit der Fachhochschule Nordwestschweiz von Peter Sommerfeld (2003) und Daniel Gredig (2005) entwickelte „Modell der kooperativen Wissensbildung“ (vgl. auch Gredig/Sommerfeld/ Kägi/Mäder/Verkuil 2003; Hollenstein/Sommerfeld/Baumgartner 2007). Dabei werden das Kollektiv und die kooperative Entwicklung von Wissen und innovativen Interventionen ins Zentrum gerückt. Praktiker und Forscherinnen arbeiten hierbei transdisziplinär zusammen, sodass das Erfahrungswissen der Praktiker in Kombination mit dem wissenschaftlichen Wissen der Forscherinnen für Innovation genutzt werden kann. Forschungsbasiert ist das Studium nicht nur dahingehend, dass das soeben erwähnte theoretische Konzept auch empirisch erhärtet wurde, sondern auch insofern, als der gesamten Konzeptentwicklung, insbesondere dem Kompetenzprofil, intern und extern durchgeführte Untersuchungen zugrunde gelegtwurden(s.a.: www.masterstudiumsozialearbeit.ch).

Das zweite Angebot, das Master-Studium „Master of Science in Social Work“ wird auf nationaler Ebene von vier Fachhochschulen gemeinsam getragen. Jede dieser vier Fachhochschulen übernimmt dabei eine Vertiefungsrichtung. Dieses Studium wird in einen Basis- und einen Vertiefungsteil unterteilt. Im Rahmen des Basisteils werden die fünf Pflichtmodule Wissenschaftstheorie, Forschungsmethoden, Theorien und Methodenentwicklung der Sozialen Arbeit, Wandel und Innovation in Sozialen Organisationen und vergleichende internationale Sozialpolitik angeboten. Im Vertiefungsteil werden vier thematische Ausrichtungen angeboten: Soziale Probleme und soziale
Systeme, Professions- und Methodenentwicklung, Sozialplanung und Sozialentwicklung sowie Sozialpolitik und Sozialökonomie (s.a.: www.masterinsozialer arbeit.ch).

\section{Fazit und Ausblick}

Will die Schweizer Fachhochschullandschaft in Sozialer Arbeit auch weiterhin international anerkannt, anschlussfähig und wettbewerbsfähig bleiben, sind die zwei dargelegten Entwicklungen erfolgreich weiterzuführen. Dies ist nicht zuletzt vor dem Hintergrund der seit 2000 stetig zunehmenden Gesamtzahl der Studierenden (Diplom bzw. Bachelor) unabdingbar, wollen sich die Fachhochschulen in Sozialer Arbeit nicht nur quantitativ, sondern auch qualitativ ausweisen. Zur Verdeutlichung: Waren es im Jahr 2000 noch 1.476 Studierende in Sozialer Arbeit, wird gemäss Bildungsprognose des Bundesamtes für Statistik (BfS 2006: S. 38) für das Jahr 2009 mit 5.300 BachelorStudierenden gerechnet.

Zur erfolgreichen Weiterführung dieser Entwicklung sind überdies Anstrengungen auf verschiedenen Ebenen nötig. Allen voran ist jede einzelne Fachhochschule gefordert, die konsequente Kompetenzorientierung nicht nur auf die Entwicklung eines Kompetenzprofils zu beschränken, sondern sie im Rahmen sämtlicher Lehrangebote konsequent einzulösen. Ferner wird von den Fachhochschulen empirisch zu belegen sein, dass die Studierenden die vorgesehenen Kompetenzen auch tatsächlich erwerben und dass die gewählten Kompetenzen für das eigene Berufsfeld und damit die eigene Profession relevant sind. Auf der Ebene Fachkonferenz gilt es auch künftig zu prüfen, welcher gemeinsamer Absprachen es zur gelingenden Umsetzung bedarf, ohne die Profilierung jeder Fachhochschule zu tangieren. Schliesslich ist auf Bundesebene die Entwicklung eines nationalen Qualifikationsrahmens für den gesamten schweizerischen Hochschulbereich voranzutreiben, der mit dem dreistufigen Qualifikationsrahmen für den europäischen Hochschulraum kompatibel ist. 